\title{
An LTS-SQUID based measurement tool for characterization of superconductive RF cavities
}

\author{
W. Vodel, R. Neubert, S. Nietzsche, K. Knaack, K. Wittenburg, and A. Peters
}

\begin{abstract}
This paper presents a new system to measure very low currents in an accelerator environment, using a Cryogenic Current Comparator (CCC). In principle a CCC is a conventional current transformer using the high performance SQUID technology to sense the magnetic fields caused by the beam current. Since the system is sensitive on a pA level, it is an optimum device to detect dark currents of superconducting cavities. The system presented here is designed for the test facilities of the superconducting accelerator modules for the European XFEL at the Deutsches Elektronen-Synchrotron (DESY) in Hamburg. Measurements in a quiet environment showed that an intrinsic noise level of the CCC of $40 \mathrm{pA} / \sqrt{ } \mathrm{Hz}$ could be achieved.
\end{abstract}

Index Terms-Accelerators, Accelerator cavities, Current comparators, SQUID.

\section{INTRODUCTION}

$\mathrm{D}^{\mathrm{s}}$ UE to the development of the TESLA technology [1] superconducting cavities have increasingly high cavity gradients. In addition to the quality factor $\mathrm{Q}$, an important criterion for good performance is a low rate of field emission. The so-called dark current consists of particles emitted by field emission, which are captured by the accelerating fields of the cavity. Since this current can be emitted at arbitrary locations in the accelerator, dark current does not fit in energy, and thus gets lost in focusing elements close to its origin. This results in additional cryogenic loss and activation of components. Therefore, dark current is often the parameter that puts practical limits on the cavity performance.

A further increase in the gradients is technically possible by developing the preparation procedures. In order to do this, or to guarantee a given performance during a larger production series, field emission or dark current has to be controlled during the fabrication process. The device presented here provides the necessary resolution and bandwidth to measure on the pA level. The CCC device takes advantage of the existing cryogenic environment; thus the requirement for cryogenic temperatures is not an additional complication.

Manuscript received August 23, 2006. This work was supported in part by the Deutsches Elektronen Synchrotron (DESY) Hamburg, Germany.

W. Vodel, R. Neubert, and S. Nietzsche are with the Friedrich Schiller University Jena, Germany (corresponding author phone: +49 3641 947421; fax: +49 3641 947422; e-mail: wolfgang.vodel@uni-jena.de).

K. Knaack, and K. Wittenburg are with DESY Hamburg, Germany. (email: klaus.knaack@desy.de).

A. Peters is with the Gesellschaft für Schwerionenforschung Darmstadt, Germany (e-mail: andreas.peters@gsi.de).
The linear accelerator technology, based on superconducting L-band $(1.3 \mathrm{GHz})$ cavities, is currently under study at DESY [1]. The XFEL will be equipped with a total of 116 superconducting acceleration modules each containing 8 "nine cell cavities". A gradient of $23.4 \mathrm{MV} / \mathrm{m}$ is required for a socalled superstructure arrangement of couples of 9-cell cavities. Higher gradients up to the physical limit at $50 \mathrm{MV} / \mathrm{m}$ are desired. The dark current, due to emission of electrons in these high gradient fields, is an unwanted particle source. Two issues are of main concern:

1) Thermal load

An emitted electron from the cavity surface follows a path along the electric field lines, and will most probably hit somewhere else inside the cavity. This leads to an additional thermal load in the cryostat, which has to be absorbed by the liquid helium refrigerator.

2) Propagating dark current

If the energy gain is sufficient, the electrons will generate secondary particles when hitting the cavity wall which in turn may also generate further secondaries. In the following avalanche process some electrons may pass through the iris of the cavity cell and will be further accelerated. In this case, if on average more than one electron passes the complete FODO (focus/defocus lattice) cell, the dark current along the LINAC will grow exponentially.

Theoretical studies [2] show that the second case seems to be the more critical one. It limits the acceptable dark current on the beam pipe "exit" of a TESLA 9-cell cavity to approximately $50 \mathrm{nA}$. Therefore the mass-production of high-gradient cavities with minimum field emission requires a precise, reliable measurement of the dark current in absolute values. The apparatus presented here senses dark currents in the nA range. It is based on the cryogenic current comparator (CCC) principle, which includes a highly sensitive LTS SQUID system as magnetic field sensor. Furthermore, the setup contains a Faraday cup and will be housed in the cryostat of the CHECHIA cavity test stand and mounted at a temperature of $1.8 \mathrm{~K}$.

\section{REQUIREMENTS FOR DARK CURRENT MEASUREMENT APPARATUS}

Electrons can leave the niobium cavity material if the force of an applied external electric field is higher than the bounding forces inside the crystal structure. The highest field gradients occur at corners, spikes or other discontinuities, due to imperfections of the cavity shape. Another potential field 


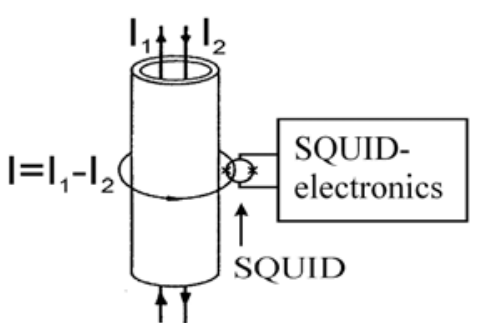

Fig. 1. Simplified scheme of a LTS SQUID-based cryogenic current comparator.

emitter is due to any kind of imperfection on the crystal matter, such as grain boundaries, inclusion of "foreign" contaminants (micro particles of e.g. In, Fe, Cr, Si, Cu) and material inhomogeneity. At these imperfections the bounding forces are reduced and electrons are emitted under the applied high electromagnetic fields [3]. The inner surfaces of the superconducting cavities undergo a series of special treatments to minimize these effects. A reliable, absolute measurement of the dark current allows the comparison of different processing methods and a quality control in the future mass-production.

The XFEL will be operated in a pulse mode with up to 10 $\mathrm{Hz}$ repetition rate. The $1.3 \mathrm{GHz}$ r.f. pulse duration is $650 \mu \mathrm{s}$ flat top. During this time the dark current is present and has to be measured. Therefore a bandwidth of $10 \mathrm{kHz}$ for the dark current instrument is sufficient. As field emission is a statistical process, the electrons leave the cavity at both ends of the beam pipe. Thus, half of the dark current exits at each side, and it has to be measured at one side only. With the $1.3 \mathrm{GHz}$ r.f. applied, we expect that the dark current will have a strong amplitude modulation at this frequency. This frequency has to be carefully rejected from the instrument electronics to insure its proper operation and to avoid a malfunction of the SQUID. This was done by the use of careful r.f. shielding, appropriate filtering of all leads feeding to the SQUID input coil, and the low pass characteristic of the transformer used.

The use of a cryogenic current comparator as dark current sensor has some important advantages:

-- measurement of the absolute value of the dark current,

-- independence of the electron trajectories,

-- accurate absolute calibration with an additional wire loop,

-- extremely high resolution.

The required working temperature of $4.2 \mathrm{~K}$ (boiling temperature of $\mathrm{LHe}$ ) for the apparatus is already provided by the CHECHIA test stand for cooling the niobium cavities. In order to enable the CCC to measure the magnetic field of the dark current only, effective shielding against external magnetic fields has to be realized.

\section{THE CRYOGENIC CURRENT COMPARATOR (CCC)}

In principle, the CCC comprises three main components (see Fig. 1):

-- the superconducting pick-up coil,

-- the highly effective superconducting shield, and

-- the high performance LTS-SQUID system.

The CCC, first developed by Harvey in 1972 [4], is a non-

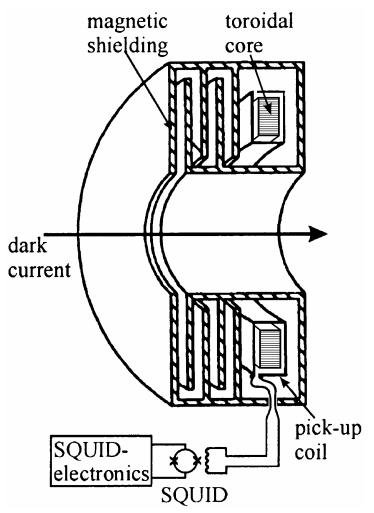

Fig. 2. Simplified schematic view of the magnetic shielding, the toroidal pick-up coil, and the SQUID.

destructive method to compare two currents $I_{1}, I_{2}$ (see Fig. 1) with high precision using a meander shaped flux transducer. Thus only the magnetic field component, which is proportional to the current in the wires, will be sensed by the pick-up coil. All other field components are strongly suppressed. In our apparatus the signal current induced in the pick-up coil is fed into the input coil of a high performance d.c. SQUID.

The design of the CCC for measurements of dark currents has been realized by collaboration between DESY Hamburg, Jena University, and GSI Darmstadt. The prototype apparatus will be placed in the CHECHIA cavity test stand and operated at a temperature of $4.2 \mathrm{~K}$.

\section{A. Pick-up Coil}

A single turn pick-up coil is formed as a superconducting niobium toroid with a slot around the circumference. It contains a Vitrovac 6025-F core (Vacuumschmelze GmbH, Hanau, Germany) providing a high permeability of about 30,000 at liquid helium temperatures [5]. According to our experience 6025-F cores give the lowest noise level in comparison to other materials tested. The material inhomogeneity of the core is averaged by complete encapsulation of a toroidal niobium coil.

\section{B. Superconductive Shields}

The resolution of the CCC is reduced if the toroidal pick-up coil operates in presence of external disturbing magnetic fields. In practice, external fields are unavoidable, therefore an extremely effective shielding has to be applied. A circular meander ("ring cavities") shielding structure (see Fig. 2) allows to pass only the azimuthally magnetic field component of the dark current, while the non-azimuthal field components are strongly attenuated.

The attenuation characteristics of CCC shields have been studied analytically in great detail [6-8]. Applied to the shielding of the TESLA CCC an attenuation factor of approximately $120 \mathrm{~dB}$ for transverse, non-azimuthal magnetic field components is estimated. This result is based on the superposition of the analytic results for the different shielding substructures, in this case coaxial cylinders and "ring cavities" (as described in detail in [9]). 


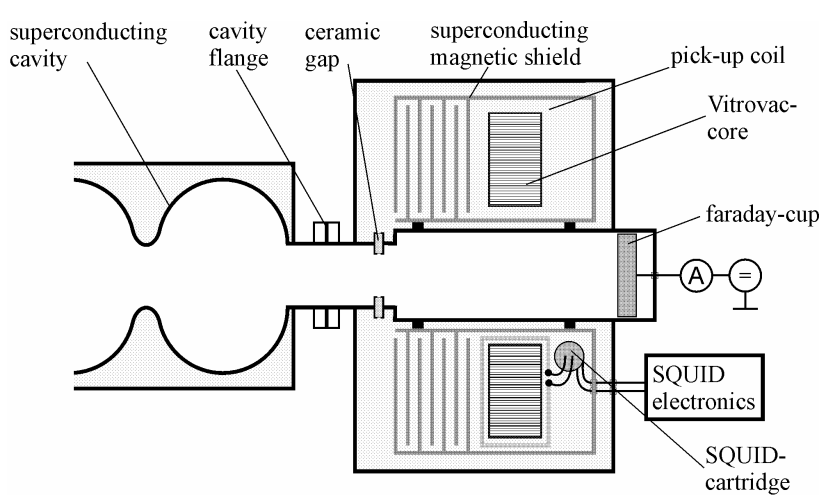

Fig. 3. Schematic view of the CHECHIA's CCC.

\section{SQUID Measurement System}

The key component of the CCC is the high performance d.c. SQUID system developed and manufactured at Jena University. The SQUID sensor UJ 111 [10] is designed in a gradiometric configuration and based on Nb-NbOx-Pb/In/Au Josephson tunnel junctions with dimensions of $3 \mu \mathrm{m} \times 3 \mu \mathrm{m}$.

The SQUID electronics consists of the low noise preamplifier and the SQUID control unit. The low source impedance of the SQUID (about $1 \Omega$ ) is stepped up to the optimal impedance of the preamplifier by a resonant transformer. The d.c. bias and flux modulation current (modulation frequency 307 $\mathrm{kHz}$ ) are fed into the SQUID via voltage-controlled current sources situated in the preamplifier and the controller, respectively. An optimal choice of bias and flux modulation point for the SQUID system was found to be a white flux spectral density of $2 \times 10^{-6} \Phi_{0} / \sqrt{\mathrm{Hz}}$. This flux noise corresponds to an equivalent current noise through the input coil of $0.9 \mathrm{pA} / \sqrt{\mathrm{Hz}}$, an effective energy factor of $543 \times h$ ( $h$ : Planck's constant), and an energy resolution of $3.6 \times 10^{-31} \mathrm{~J} / \mathrm{Hz}$ [10].

In a d.c. coupled feedback loop, the field of the dark current to be measured is compensated at the SQUID by an external magnetic field generated by the attached electronics. Due to the superconductivity of all leads in the input circuitry (pickup coil, transformer, SQUID input coil) the CCC is able to detect even d.c. currents. For an optimum coupling between

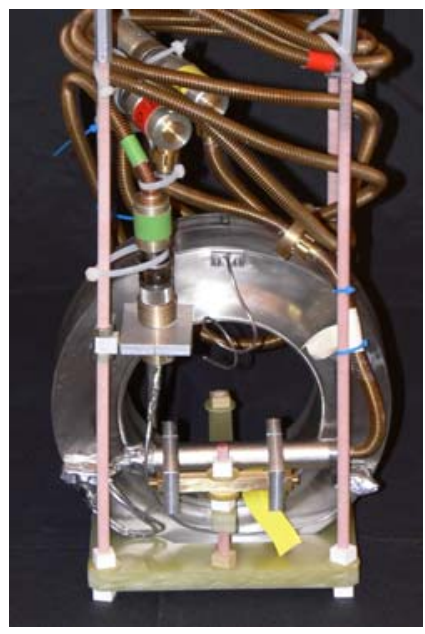

Fig. 4. Nb pick-up coil prepared for preliminary tests at 4.2 $\mathrm{K}$ in a liquid helium wide-neck cryostat.

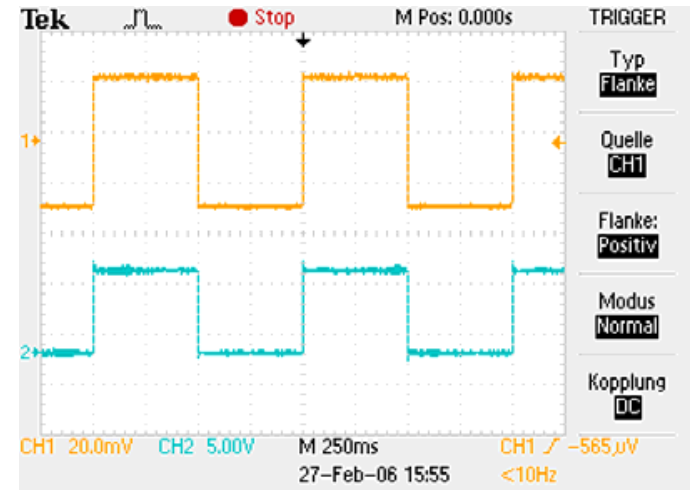

Fig. 5. Calibration signal (upper curve) and SQUID output signal (lower curve). See text for details.

the 1-turn toroidal pick-up coil $(40 \mu \mathrm{H})$ and the SQUID input a matching transformer is necessary. The overall current sensitivity of the CCC was calculated to be $175 \mathrm{nA} / \Phi_{0}$.

Using a modulation frequency of $307 \mathrm{kHz}$ the measurement system provides an over-all bandwidth of $20 \mathrm{kHz}$ (signal level $1 \Phi_{0}$ ) or $70 \mathrm{kHz}$ (signal level $0.1 \Phi_{0}$ ). Thus, it will be possible to characterize the pulse shape of the dark current beam (300 $\mu$ s rise time, $950 \mu$ s flattop, $300 \mu$ f fall time, $10 \mathrm{~Hz}$ repetition rate) which is dominated by the r.f. structure applied to the cavities (see also Fig. 6).

\section{Faraday Cup}

Because the energy of dark current electrons is relatively small at CHECHIA, the design includes a Faraday cup to provide a second measurement system for comparison. We installed the Faraday cup at the end of the cavity vacuum chamber. The readout electronics will measure the current to ground.

The simplified scheme of the main components of the CHECHIA's CCC is shown in Fig. 3. Later configurations for complete module tests in the test stand will not allow simple Faraday cups because of the much higher energy (some 100 $\mathrm{MeV}$ ) of the dark current.

\section{RESULTS}

Test measurements of the completed pick-up coil with the special cabling and feed-throughs at $4.2 \mathrm{~K}$ were successfully carried out in a wide-neck cryostat at the cryogenic laboratory of Jena University (see Fig. 4). A current generator was used as a signal source to simulate the expected dark electron beam pulses. By supplying the calibration coil with a calibrated current pulse the current sensitivity of the CCC was found to be $200 \mathrm{nA} / \Phi_{0}$, which is in a good agreement with the design value of $175 \mathrm{nA} / \Phi_{0}$.

Fig. 5 shows the SQUID output signal with an amplitude of $0.85 \Phi_{0}$ (lower curve) and the test pulse signal of $170 \mathrm{nA}$ (upper curve) with a repetition rate of $1 \mathrm{~Hz}$. 


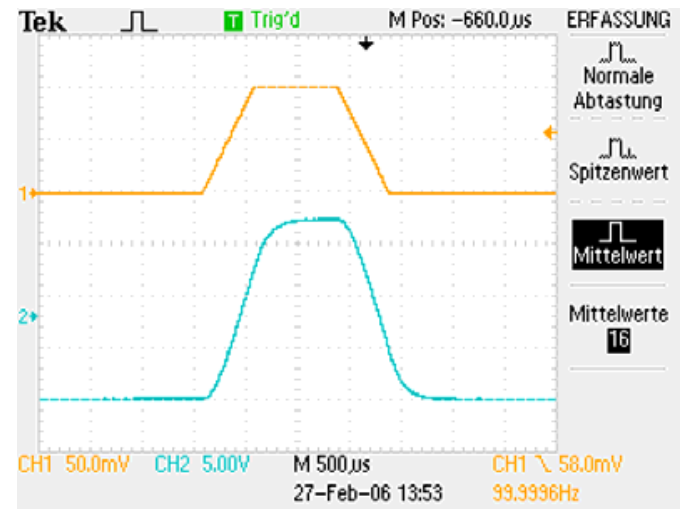

Fig. 6. Simulated beam pulse (upper curve, $300 \mu$ sise time, $950 \mu$ s flattop, $300 \mu$ s fall time) and SQUID output signal (lower curve).

Fig. 6 shows of plot of the simulated beam signal generated by a programmable current generator with the amplitude of $340 \mathrm{nA}$. The response amplitude of the SQUID system was $1.7 \Phi_{0}$ corresponding with a current sensitivity of $200 \mathrm{nA} / \Phi_{0}$.

The spectral flux noise density of the system in the frequency range between 1 and $1600 \mathrm{~Hz}$ was measured using a HP spectrum analyzer in the laboratory at Jena University and a level of $2 \times 10^{-4} \Phi_{0} / \sqrt{ } \mathrm{Hz}$ was observed (see Fig. 7). For these measurements a low pass filter with a cut-off frequency of 1 $\mathrm{kHz}$ was used. This flux noise level corresponds to a noise limited current resolution of the system of $40 \mathrm{pA} / \sqrt{\mathrm{Hz}}$ which is significantly better than required. As a result of the rough measurement conditions at DESY a noise limited current resolution of the CCC of $500 \mathrm{pA} / \sqrt{\mathrm{Hz}}$ was achieved. This is primarily a result of the external electrical disturbances and mechanical vibrations caused by numerous machines and other equipment of the test facility.

Long-term measurements of the output voltage of the CCC to detect flux drifts, caused for instance by temperature drifts of the core material (Vitrovac 6025-F), showed a sufficient small drift of $<2 \times 10^{-5} \Phi_{0} / \mathrm{s}$.

\section{CONCLUSION}

The mechanical construction of the CHECHIA CCC is complete. Tests of the manufacturing of critical components, above all the meander shaped niobium shielding and the CCC's housing, were successfully carried out. The SQUID electronics including special cabling and feed throughs are ready for installation at DESY.

The completed pick-up coil with connected SQUID system were tested in a wide-neck LHe cryostat at $4.2 \mathrm{~K}$. Supplying the calibration coil with a calibrated current pulse the current system sensitivity of the CCC could be measured and a value of $200 \mathrm{nA} / \Phi_{0}$ was found.

In spite of the rough measurement conditions at DESY a noise limited current resolution of the CCC of $500 \mathrm{pA} / \sqrt{\mathrm{Hz}}$ was achieved. Measurements in a quiet environment in the low temperature laboratory at Jena University showed that this value could be improved by more than one order of magnitude

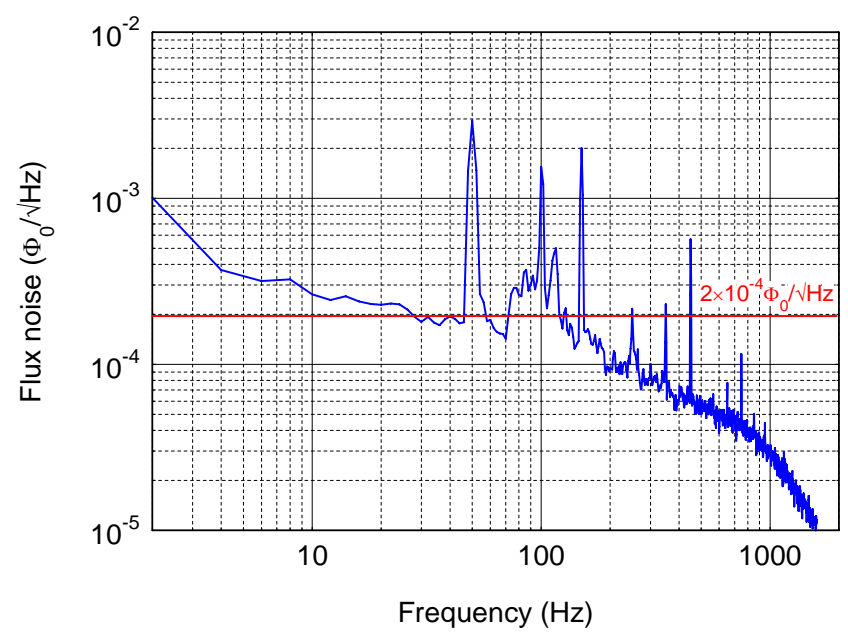

Fig. 7. Noise spectrum of the CCC within a bandwidth from $1 \mathrm{~Hz}$ to $1600 \mathrm{~Hz}$. Low pass filter: $1 \mathrm{kHz}$.

down to a noise level of $40 \mathrm{pA} / \sqrt{ } \mathrm{Hz}$.

Long-term measurements of the output voltage of the CCC showed that the drift of the whole system is negligible small and appears in the range of $<2 \times 10^{-5} \Phi_{0} / \mathrm{s}$.

The complete CCC is now ready for use and the final commissioning of the apparatus in the CHECHIA test stand is planned within the next months. The start of the dark electron beam measurements to qualify the superconductive accelerator r.f. cavities is planned for spring 2007.

\section{ACKNOWLEDGMENT}

The authors wish to thank R. Nawrodt for his support in performing measurements and computer work. We also thank L. Saenger as well as the company pro-beam AG, Burg, Germany, for their expert assistance in manufacturing most of the mechanical parts for the CCC. We highly appreciate I. Martin for helpful discussions.

\section{REFERENCES}

[1] R. Brinkmann, TESLA Technical Design Report.

[2] R. Brinkmann, Dark Current Issues; TESLA Collab. Meeting - CEA Saclay, April 2002.

[3] G. R. Werner et al., Proc. of the Part. Acc. Conf. PAC 01, pp. 1071-73, 2001.

[4] I. K. Harvey, “A precise low temperature dc ratio transformer”, Rev. Sci. Instrum., vol. 43, p. 1626, 1972.

[5] Datasheet "Toroidal strip-wound cores of VITROVAC 6025 F" and private communication from Vacuumschmelze Hanau, Germany, 2002.

[6] K. Grohmann et. al., CRYOGENICS, pp. 423-429, July 1976.

[7] K. Grohmann et. al., CRYOGENICS, pp. 601-605, October 1976.

[8] K. Grohmann, D. Hechtfischer, CRYOGENICS, pp. 579-581, October 1977.

[9] P. Gutmann, H. Bachmair, in V. Kose, Superconducting Quantum Electronics, pp. 255-259, 1989.

[10] W. Vodel, K. Mäkiniemi, “An ultra low noise SQUID system for biomagnetic research”, Measurement Science and Technology, Vol 3, No 2, pp. 1155-1160, Dec. 1992. 